\title{
Argon Plasma Coagulation
}

National Cancer Institute

\section{Source}

National Cancer Institute. Argon Plasma Coagulation. NCI Thesaurus. Code C66714.

A non-contact form of electrocoagulation in which high-frequency electric current is delivered via ionized argon gas to cause coagulation and destruction of tissue. 